\title{
Distribution of Enhancement of the Electric Field of Plane Wave Laser around Gold Tip Optical Antenna
}

Mehdi Zohrabi ${ }^{*}$

Department of Optics and Nanophotonics, Institute of Physics, Kazan Federal University, Russia

\begin{abstract}
This paper provides a new design of gold tip optical antenna based on a specific geometry, and then the change of enhancement of the electric field of plane wave laser excitation with 400 to $700 \mathrm{~nm}$ in the around of optical antenna are simulated. Changing the geometrical of light antenna includes of creating circular gratings with the period, 200,300 nm on the shaft of antenna. With the exerting of laser light to the place of these gratings .the distribution of enhancement of the electric field in a plane perpendicular to the shaft has been acquired. Finally, the optimized value for the maximum enhancement at the period of $386.102 \mathrm{~nm}$ is obtained.
\end{abstract}

Keywords: Optical antenna; Gold tip; Field enhancement; Surface plasmon; Localized plasmon

\section{Introduction}

Optical antennas are devices which task is effective transformation of radiation in the optical range in localized and back. Optical antennas can take various unusual forms (probes, nanoparticles, etc.), and their properties strongly depend on a form and material owing to superficial the plasmon of resonances. The receiver or the transmitter interacts with free optical radiation by means of the optical antenna..The receiver or the transmitter usually elementary quantum absorber or a radiator, such as atom, an ion, a molecule, a quantum point, or the defective center in a solid body. The antenna strengthens interaction between a radiator or an absorber. Therefore it provides prospect to control interaction of light with substance at the level of one quantum system. Existence of the antenna changes properties of the receiver/transmitter, for example rates of transition and, in case of strong interaction, even structure of power levels. Besides, properties of the antenna depends on properties of the receiver transmitter, and becomes obvious that their two it is necessary to consider as the related system. Properties of optical .antennas are defined by behavior of electronic plasma in limited metal nanostructures. These properties depend on many parameters: form and size of the nanoantenna, material, crystallographic orientation, polarization of laser radiation, wavelength of an exciting field, light hade. The variation of these parameters allows "to adjust" system of resonances on effective interaction of light with nanodimensional system [1]. It is reached, by change of physical characteristics of optical antennas. The main characteristics are the local density of electromagnetic states, the disseminated power and an impedance of the antenna, efficiency, an orientation and strengthening of radiation, an aperture of the antenna and section of absorption.

By utilizing optical antenna, it is possible to concentrate the energy of the laser in a little scale zone such as nanometer. Gold tip is one of the most popular sort of optical antenna in the recent years [2]. One of the advantages of this antenna is the break of the limit of diffraction. The enhancement in the electrical field around the gold tip as a result of the plasmon resonance, which is depending on the antenna geometry and the impact of lightning rod effect [5]. Direct radiation of the tip apex, in the NSOM prompted creating of the foundation signal and impress the efficiency of the antenna and numerous exploration have been carried out to reduce this issue [4]. Adiabatic Nano focusing along conical metal tapers describes a coherent transport of optical excitations in the form of surface plasmon polariton (SPP) waves over several tens of $\mu \mathrm{m}$ and the concentration of this energy into a nanometric volume at the taper apex. In the adiabatic limit, i.e., if the waveguide cross section variation is slow and relative changes of the SPP wave vector are small on a scale of the SPP wavelength, radiative and reflective losses are minimized and energy transport to the apex is expected to be particularly efficient [3].

Raschke et al [6] proposed the concept of adiabatic nanofocusing, in this method by reducing the size of the region in which enhancement of electric field occurs, the background might be diminished mostly. In the adiabatic nanofocusing method, first the surface plamson of the shaft is excited. Then the surface Plasmon to the apex of tip is travelled and finally is convert to localized plasmon in the apex. In this paper, the geometry of the ordinary gold tip have been changed. Another work, adiabatic nanofocus based the Raman scattering experiment. Electrochemically etched Au tips are mounted onto the quartz tuning fork of a shear-force AFM, and a grating is cut using FIB. Incident light is focused onto the grating, and the Raman scattered light excited by the nanofocused SPP at the apex is detected at a 90-deg angl.in this work enhancement of Raman scattering as a result of grating coupling was shown [7]. Adiabatic nano-focusing is an efficient method of converting far-field optical signal into near-field localized light source [8], and has immense potential to perform background-free nanoscale chemical spectroscopy and imaging.[9-10]. all of these work are experimentally done and the role of gratting on the result were known well but theoretically until now nobody can't explain the role of gratting on the distribution of enhancement and mostly it is done by method of simulation with different softwares.in this work we designed a optical antenna with special geometry.

Moreover, circular gratings with a period of 200 and $300 \mathrm{~nm}$ has been included. Maxwell equations with FDTD simulation numerical software have been utilized to gain the results. In this geometry, different peak as a result of excitation of surface plasmon of grating have been obtained.

*Corresponding author: Mehdi Zohrabi, Department of Optics and Nanophotonics, Institute of Physics, Kazan Federal University, 18 kremlyovskaya St, kazan 420008 Russia, Tel: 843-233-71-09; E-mail: zh_als@yahoo.com

Received October 29, 2014; Accepted November 18, 2014; Published November 26, 2014

Citation: Zohrabi M (2014) Distribution of Enhancement of the Electric Field of Plane Wave Laser around Gold Tip Optical Antenna. J Nanomed Nanotechnol 5 245. doi: 10.4172/2157-7439.1000245

Copyright: @ 2014 Zohrabi M. This is an open-access article distributed under the terms of the Creative Commons Attribution License, which permits unrestricted use, distribution, and reproduction in any medium, provided the original author and source are credited. 
Citation: Zohrabi M (2014) Distribution of Enhancement of the Electric Field of Plane Wave Laser around Gold Tip Optical Antenna. J Nanomed Nanotechnol 5: 245. doi: 10.4172/2157-7439.1000245

Page 2 of 5

\section{Discussion}

The Finite-Difference Time-Domain method (FDTD) is today's one of the most popular technique for the solution of electromagnetic problems. It has been successfully applied to an extremely wide variety of problems, such as scattering from metal objects and dielectrics, antennas, microstrip circuits, and electromagnetic absorption in the human body exposed to radiation. The main reason of the success of the FDTD method resides in the fact that the method itself is extremely simple, even for programming a three-dimensional code. The technique was first proposed by $\mathrm{K}$. Yee, and then improved by others in the early 70s. The theory on the basis of the FDTD method is simple. To solve an electromagnetic problem, the idea is to simply discretize, both in time and space, the Maxwell's equations with central difference approximations. The originality of the idea of Yee resides in the allocation in space of the electric and magnetic field components, and the marching in time for the evolution of the procedure. Only the electric field component is chosen to evaluate the enhancement.

To generate a strong field enhancement at the tip, the electric field of the exciting laser beam needs to be polarized along the tip axis. The influence of tip shape and material on the field enhancement has been

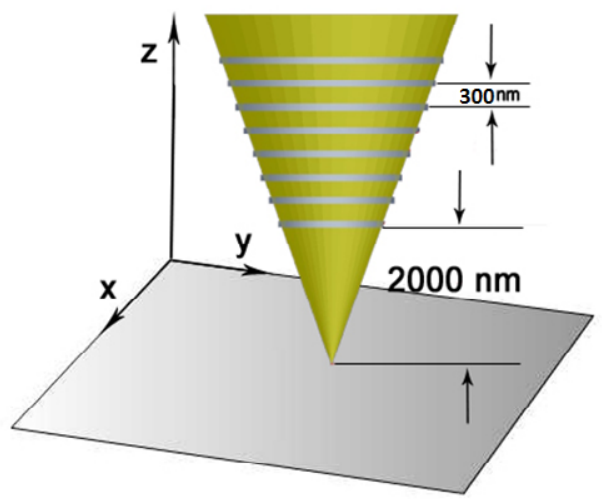

Figure 1: Considered shape for optical antenna

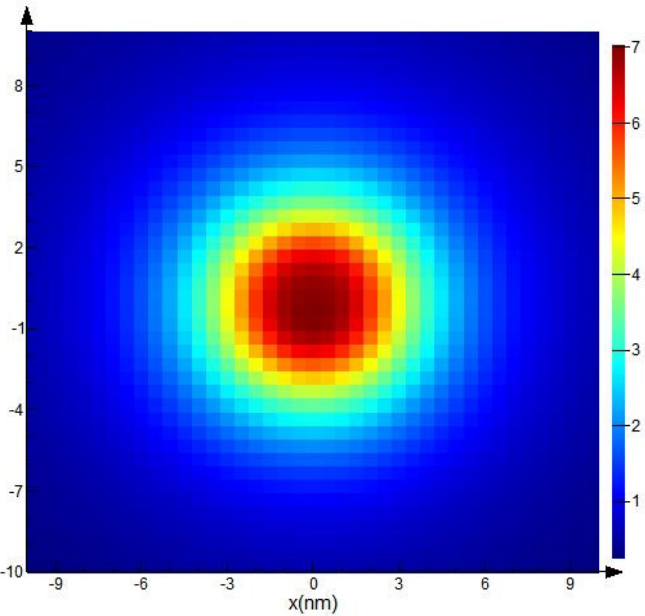

discussed in a series of publications with the expect to discover the optimum tip.

The electric filed around the optical antenna is calculated and simulated based on Maxwell's equations.

$$
E(r)=E_{0}+i \omega \mu \mu_{0} \int_{v} \vec{G}\left(r, r^{\prime}\right) j\left(r^{\prime}\right) d V^{\prime}
$$

Where $\vec{G}$ is the dyadic Green's function and $E_{0}$ represents the initial electric field for the plane wave laser [11]. In the case of Raman scattering, the total signal depends on the product of the transition rates $k_{e x}\left(\lambda_{e x}\right) k_{\text {rad }}\left(\lambda_{\text {rad }}\right)$. As a consequence, the total signal enhancement scales with the fourth power of the field enhancement for small differences between the excitation and emission wavelength, assuming that the field enhancement at the tip does not depend sensitively on the wavelength [Eq. (3)].

$$
\begin{aligned}
& f=\left(\frac{E}{E_{0}}\right) \\
& M_{\text {Raman }}=\left(k_{\text {ex }, \text { tip }} / k_{\text {ex }, 0}\right)\left(k_{\text {rad,tip }} / k_{\text {rad }, 0}\right) \approx f^{2}
\end{aligned}
$$

For the general case of surface-enhanced Raman scattering (SERS), enhancement factors of up to 12 orders of magnitude have been reported for particular multiple particle arrangements with interstitial sites between the particles or sharp protrusions on the outside surface [12].

\section{Simulation results}

In this work, we investigated the influence of tip shape on the field enhancement. We assumed a golden tip, a 10 - nm radius of apex and a $35^{\circ}$ Cone angle and two proposed period of grating which are 200 and $300 \mathrm{~nm}$. The laser beam is considered as a plane wave with the wavelength of $400-700 \mathrm{~nm}$. The considered model and the simulation results for the considering grating period is shown in following. Figure 1 illustrates the shape of proposed optical antenna with $300 \mathrm{~nm}$ period of grating.

Figures 2 show the intensity distribution for three proposed period of grating with 90 degree laser incident angle in $\mathrm{X}$ and $\mathrm{Y}$ axis.

These figures confirm that there are significant changes in intensity distribution in the plane of $\mathrm{X}-\mathrm{Y}$ where $\mathrm{Z}=0 \mathrm{~nm}$.

Figure 2: a) Intensity distribution with $200 \mathrm{~nm}$ period of grating. b) Intensity distribution with $300 \mathrm{~nm}$ period of grating. 
(a)

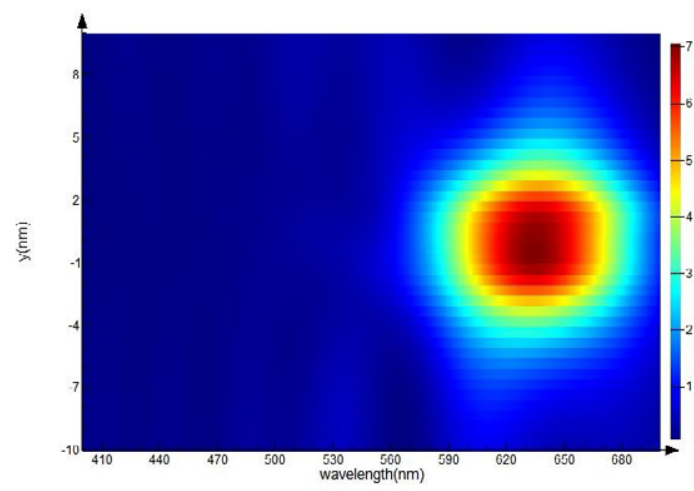

(c)

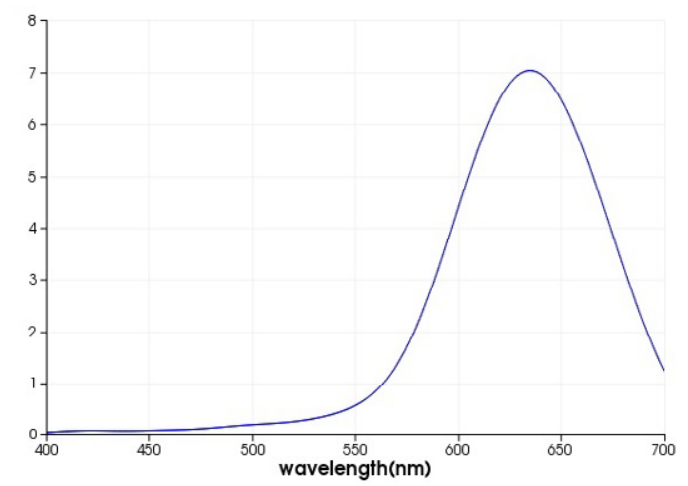

(d)
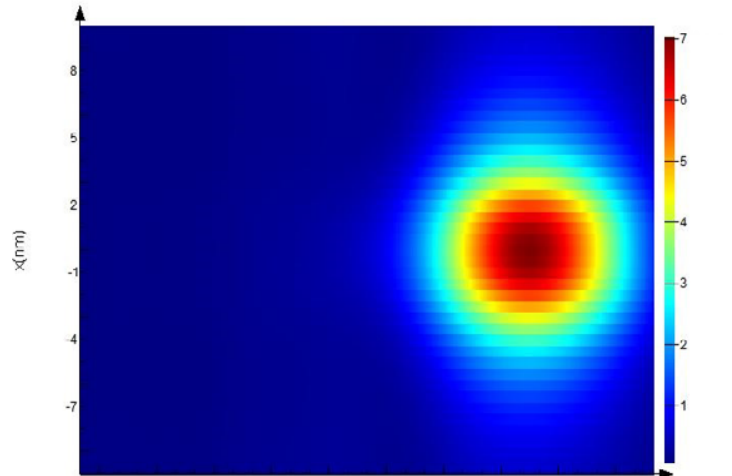

Figure 3: (a) the distribution intensity with $y$ on the line of $x=0, z=0$ (b) the curve of enhancement of $y$ component of electric field in $y=0$ versus wavelength (c) the intensity distribution intensity with an $x$ on the line of $y=0, z=0$ (d) the curve of enhancement of the $x$ component of electric field in $x=0$ versus wavelength.

(a)

(b)
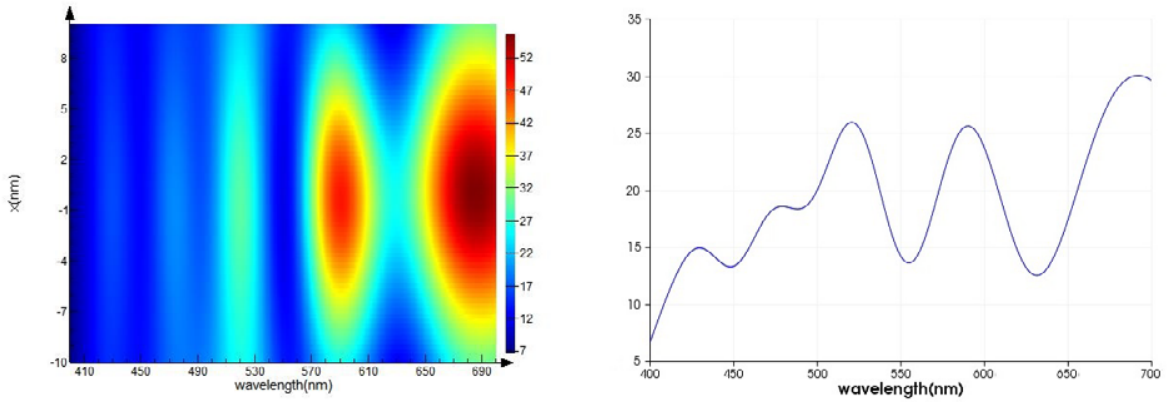

(c)

(d)
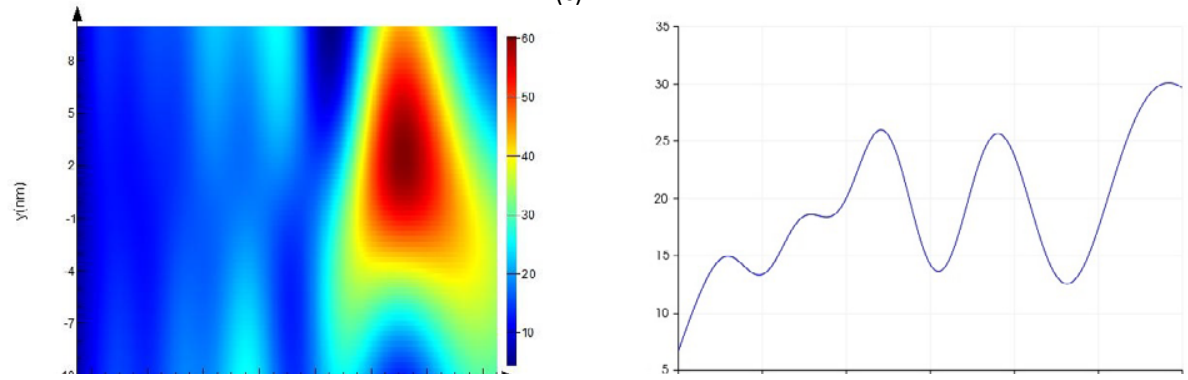

Figure 4: (a) the distribution intensity with $y$ on the line of $x=0, z=0$; (b) the curve of enhancement of $y$ component of electric field in $y=0$ versus wavelength; (c) the intensity distribution intensity with an $x$ on the line of $y=0, z=0$ (d) the curve of enhancement of the $x$ component of electric field in $x=0$ versus wavelength. 


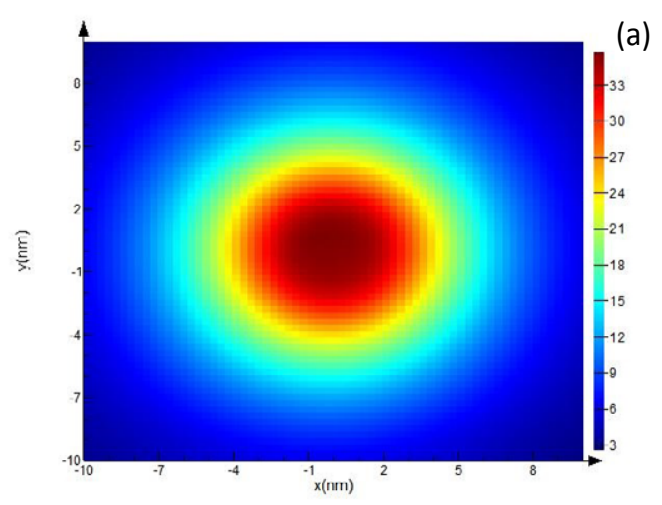

(b)

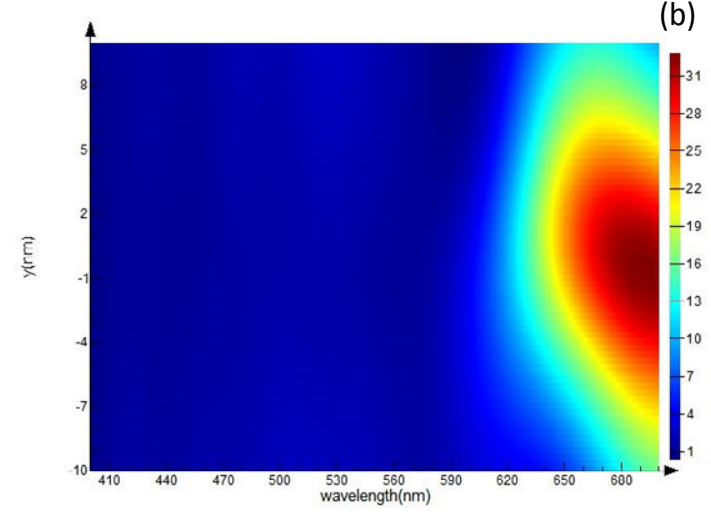

(d)

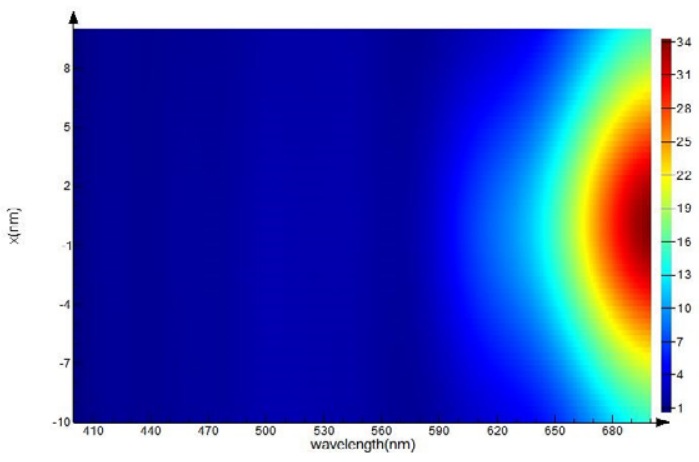

(a)

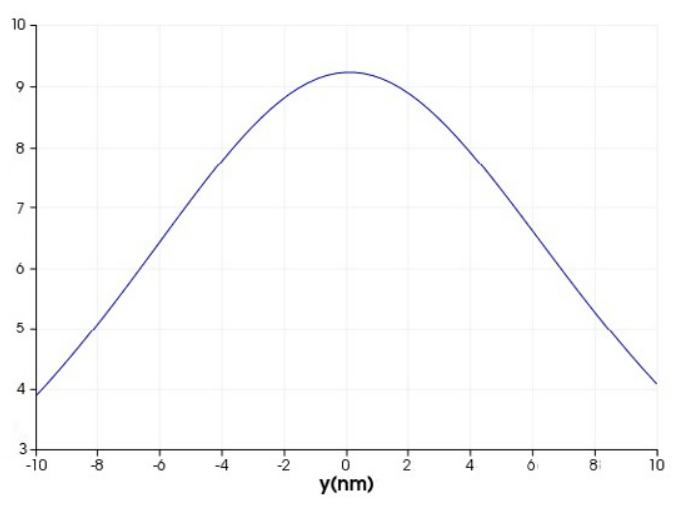

(f)

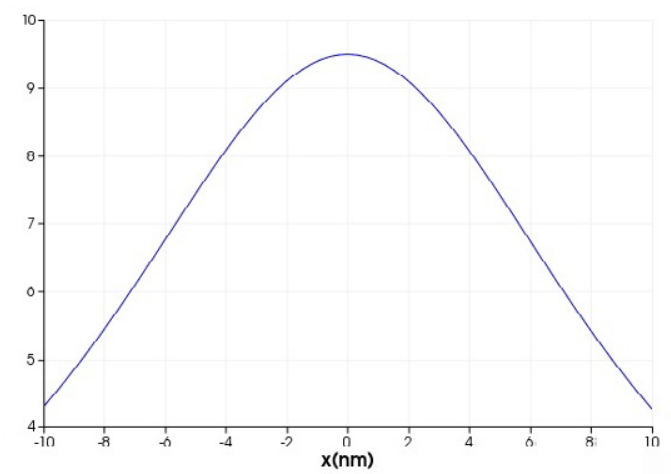

Figure 5: Intensity distribution in simulation result when the period of the grating is $208.843 \mathrm{~nm}$ (optimized) with 90 degree laser incident angle. (a) the intensity distribution in the plane of $x-y(z=0 \mathrm{~nm})$, (b) the distribution intensity with $y$ on the line of $x=0, z=0$ (c) the curve of enhancement of $y$ component of electric field in $y=0$ versus wavelength (d) the intensity distribution intensity with an $x$ on the line of $y=0, z=0$ (e) the curve of enhancement of the $x$ component of electric field in $\mathrm{x}=0$ versus wavelength.

Figures 3 to 5 are represented the distribution intensity in $\mathrm{X}$ and $\mathrm{Y}$ directions for three proposed period of grating. Moreover, the curves of enhancement of $\mathrm{X}$ and $\mathrm{Y}$ component of electric field versus their wavelength are depicted.

According to the simulation results,each peak of these graphs is as a result of excitation of surface Plasmon of grating on the shaft of antenna and can be concluded that by changing the period of grating on the shaft, the resonance can be shifted which results in changing the distribution of enhancement of the electric field.

\section{Maximize the electric field enhancement}

The obtaining of the maximum enhancement in the electric field is an important fact and can be utilize for the optimum design of the antenna. The optimized value for the period of grating based on the maximum electric field enhancement equals to $386.102 \mathrm{~nm}$.

The results of the optimization and maximum enhancement of the electric field for the new period of grating are illustrated in figure 4 . 
Citation: Zohrabi M (2014) Distribution of Enhancement of the Electric Field of Plane Wave Laser around Gold Tip Optical Antenna. J Nanomed Nanotechnol 5: 245. doi: 10.4172/2157-7439.1000245

Page 5 of 5

\section{Conclusion}

In this work, to calculate the enhancement of electric field intensity in the near - field area, the FDTD algorithm is applied. To sum up, the enhancement of the near-field the Apertureless probe system has been testified. The proposed gold tip optical antenna has special geometry and in this geometry, a series of circular grating added to the shaft. The simulated model shows, with the change in the period of grating on the shaft, the resonance can be shifted and this led to changing of the distribution of enhancement of the electric field. Changes of geometry include change of period of circular grating in the interval of 200-300 $\mathrm{nm}$, on the shaft of the antenna. Distribution of enhancement of the electric field in a plane perpendicular to the shaft for the three samples has been analyzed. In addition, It is investigated that the maximum enhancement of the electric field accrues for the period of $386.102 \mathrm{~nm}$.

\section{Acknowledgment}

The authors wish to thank prof. M.KH.Salakhov, for helpful discussions. We are also grateful Department of Optics and Nano photonics, Kazan Federal University to for giving us access to their equipment.

\section{References}

1. De Angelis F, Das G, Candeloro P, Patrini M, Galli M, et al. (2010) Nanoscale chemical mapping using three-dimensional adiabatic compression of surface plasmon polaritons. Nat Nanotechnol 5: 67-72.

2. Behr N, Raschke MB (2008) Optical antenna properties of scanning probe tips: plasmonic light scattering, tip-sample coupling, and near-field enhancement. The Journal of Physical Chemistry C 112: 3766-3773.
3. Giannini V, Sánchez-Gil JA (2008) Excitation and emission enhancement of single molecule fluorescence through multiple surface-plasmon resonances on metal trimer nanoantennas. Opt Lett 33: 899-901.

4. Hartschuh A (2008) Tip-enhanced near-field optical microscopy. Angew Chem Int Ed Engl 47: 8178-8191.

5. Liao PF, Wokaun A (1982) Lightning rod effect in surface enhanced Raman scattering. The Journal of Chemical Physics 76: 751-752.

6. Berweger S, Atkin JM, Olmon RL, Raschke MB (2010) Adiabatic tip-plasmon focusing for nano-Raman Spectroscopy. J Phys Chem Lett 24: 3427-3432.

7. Berweger S, Atkin JM, Olmon RL, Rashke MB (2010) Adiabatic tip-plasmon focusing for nano-Raman spectroscopy. J Phys Chem Lett 1: 3427-3432.

8. Stockman MI (2004) Nanofocusing of optical energy in tapered plasmonic waveguides. Phys Rev Lett 93: 137404.

9. Neacsu CC, Berweger S, Olmon RL, Saraf LV, Ropers C, et al. (2010) Nearfield localization in plasmonic superfocusing: a nanoemitter on a tip. Nano Lett 10: 592-596.

10. Ropers C, Neacsu CC, Elsaesser T, Albrecht M, Raschke MB, et al. (2007) Grating-coupling of surface plasmons onto metallic tips: a nanoconfined light source. Nano Lett 7: 2784-2788.

11. Novotny L, Hecht B (2012) Principles of Nano-optics. $2^{\text {nd }}$ edtn, Cambridge university press, UK.

12. Mauser N, Achim H (2014) Tip-enhanced near-field optical microscopy. Chemical Society reviews 43: 1248-1262. 